\title{
Unexpected schwannoma unmasked by the physiologic changes of pregnancy
}

\author{
Marijn C.T. Tacken ${ }^{*}$, Bart P. Ramakers ${ }^{1,2}$, Albert J.S. Idema ${ }^{3}$ and Johannes G. Van der Hoeven ${ }^{1,4}$ \\ ${ }^{1}$ Department of Intensive Care, Radboud University Medical Centre, Nijmegen, the Netherlands \\ ${ }^{2}$ Department of Intensive Care, Bernhoven Hospital, Uden, the Netherlands \\ ${ }^{3}$ Department of Neurosurgery, Radboud University Medical Centre, Nijmegen, the Netherlands \\ ${ }^{4}$ Head of Department of Intensive Care, Radboud University Medical Centre, Nijmegen, the Netherlands
}

\begin{abstract}
A 35-year-old pregnant women at 36 weeks gestation was admitted to the department of neurology because of a rapidly progressive pyramidal syndrome with paresis of her arms and legs. Analysis revealed an extensive schwannoma with spinal cord compression for which she received a hemilaminectomy. This case illustrates how the physiologic changes during pregnancy (as hypervolemia) may reveal a rare tumor.
\end{abstract}

\section{Introduction}

A schwannoma is a benign tumor of the nerve sheath, typically occurring as a solitary, encapsulated mass of cranial nerves, peripheral nerves or spinal roots. These tumors are usually slow growing and often fill a significant volume of the spinal column before causing symptoms. Most commonly they present with radicular sensory changes and pain. We report a young patient with an extensive schwannoma possibly unmasked by the normal physiologic changes of pregnancy.

\section{Case report}

A 35-year-old pregnant women at 36 weeks gestation was admitted to the neurology department because of a pyramidal syndrome with paresis of her arms and legs. Simultaneously there were progressive pain symptoms of her neck that had been present for many years. A MRI of the spine showed a space engaging process from $\mathrm{C} 3$ to T7 (Figure 1). Soon after admission the patient became hypoxic and therefore she was intubated and admitted to the intensive care unit. Because of the threatening spinal cord compression a caesarean section was performed the day after admission. A healthy girl baby was born. Shortly after the operation the patient could be extubated. On day 3 a diagnostic hemilaminectomy was performed. Histological examination showed a schwannoma. On day 10 a hemilaminectomy of $\mathrm{C} 3$ to $\mathrm{T} 7$ was performed and the tumor was resected in total (Figure 2). During the operation the root of $\mathrm{C7}$ on the left side was cut. The postoperative course was uneventful and she was dismissed to the neurosurgery ward on day 11 . Neurologic function slowly recovered. Two weeks later she was discharged to a rehabilitation centre. Seven months after admission the patient has almost completely recovered except for decreased strength and tingling of her left hand. A control MRI showed no residual tumor (Figure 2).

\section{Discussion}

We report a patient with a probably long standing schwannoma with worsening complaints elicited by the normal physiology changes of pregnancy. It has been suggested that hypervolaemia, combined with obstruction of the inferior vena cava by the enlarging uterus could result in engorgement of the venous system, especially the extradural veins distal to the occluded zone [1]. Because of the combination of the tumor and hypervolamia with the engorgement of the venous system the occlusion of the spinal canal may result in hypoxia and metabolic disturbances of unmyelinated nerves, leading to back pain or in worse case to paraplegia.

Schwannomas are peripheral nerve sheath tumors comprised entirely of neoplastic Schwann cells. They belong to the group of nerve sheath tumors which may be either sporadic or associated with an inherited disorder (neurofibromatosis, familial schwannomatosis). The incidence of sporadic spinal schwannoma is $0.3 / 100.000$ (women) and $0.4 / 100.000$ (men) [2].

The majority of nerve sheath tumors are confined to the intradural, extramedullary space, but some of these tumors extend into either the extradural compartment or the spinal cord. Schwannomas are typically situated eccentrically to the nerve. Occasionally spinal nerve sheath tumors grow intramedullary. Schwannomas are considered benign tumors, although malignant subtypes exist. Of all intradural extramedullary localized tumors $25 \%$ are nerve sheath tumors, of which about $65 \%$ is a schwannoma [3].

Schwannomas are typically slow growing and often fill a significant volume of the spinal column before causing symptoms. The specific symptoms of nerve sheath tumors depend upon the level of the spinal cord involved. They commonly present with pain, which is typically worse at night or in the morning and resolves during the

Correspondence to: Marijn C.T. Tacken, Department of Intensive Care, Radboud University Medical Centre, Nijmegen, the Netherlands, E-mail: Marijn.Tacken@radboudumc.nl

Key words: pregnancy, schwannoma

Received: July 11, 2016; Accepted: July 26, 2016; Published: July 29, 2016 


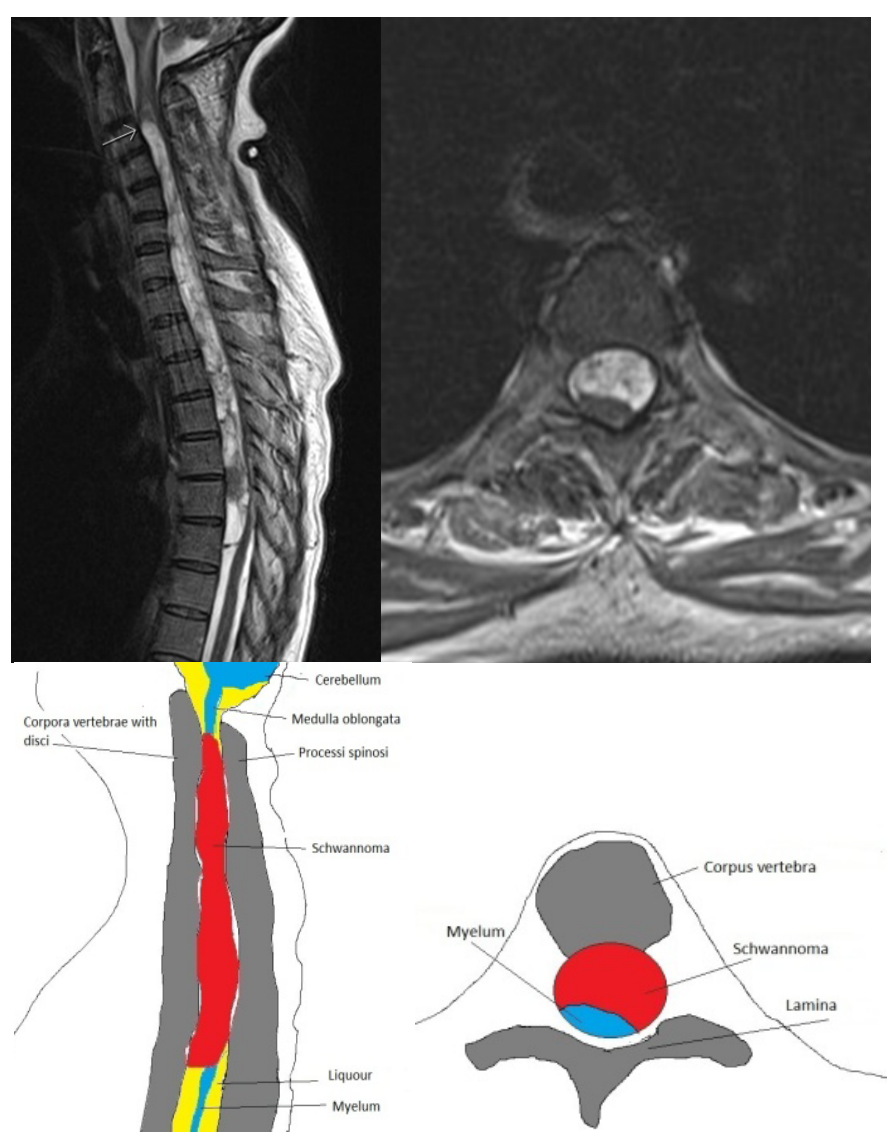

Figure 1. MRI left sagittal section thoracic spine (arrow: upper limit of schwannoma) and right: sagittal section at the height of thoracic 4 pre-operative.

day. Schwannomas usually arise from the dorsal sensory roots and may present with radicular sensory changes. Once a tumor reaches a critical mass and causes spinal cord compression, signs and symptoms of myeolopathy can progress rapidly [4].

\section{Treatment}

If asymptomatic, schwannomas may be followed with serial MRI given their usual benign behavior. Symptomatic or radiographiclly enlarging tumors should undergo maximal safe resection [5]. The treatment for intradural extramedullary schwannoma is surgery [6]. The main part of all intradural spinal schwannomas can be removed by the posterior or posteriorlateral approach. Often it is sufficient with a unilateral hemilaminectomy. Depending on the type of tumor sometimes this must be supplemented by partial or total facetectomy.

With an intraspinal ventrally located tumor across multiple segments often an anterior approach combined with a (partial) corporectomy and fusion is necessary [7-9]. In this case a combined posterior and anterior approach was planned. Per-operatively it appeared that the tumor in total could be resected by the posterior approach by means of a hemilaminectomy.

The clinical outcome after removal of a spinal schwannoma correlates closely to the severity of the preoperative symptoms. In a study including 187 patients with a schwannoma [4], all tumors were surgical removed, and 11 patients had recurrence of disease. Only two patients were re-operated. The perioperative mortality rate was $6 \%$ ( $1 \%$ during the operation and $5 \%$ through a late complication). A few

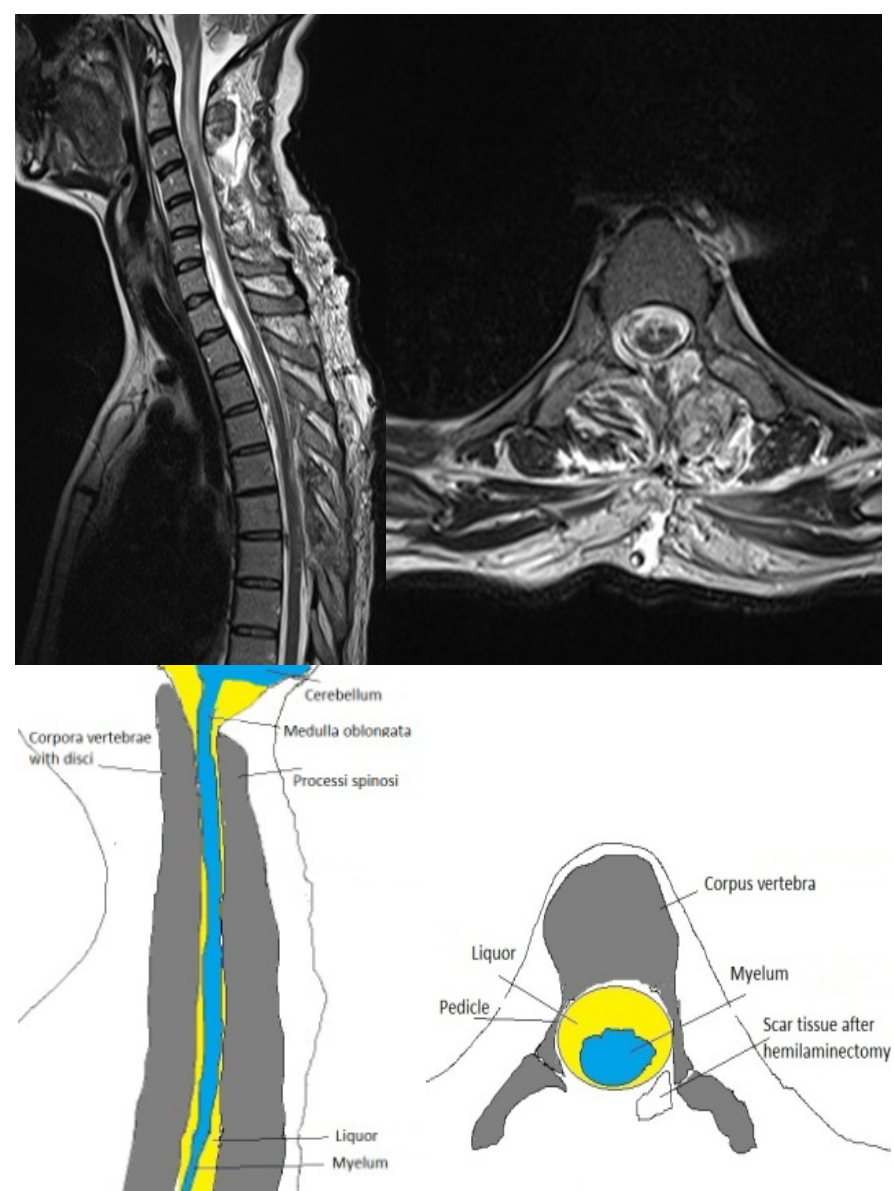

Figure 2. MRI left sagittal section thoracic spine and right sagittal section at the height of thoracic 4 post-operative.

months after the operation $78 \%$ of patients were improved, $15 \%$ were in the same condition and $7 \%$ were worse than pre-operatively.

The life expectancy of the patients equals that of the corresponding general population [4].

\section{Conclusion}

This case illustrates how the physiologic changes during pregnancy may unmask a long-standing schwannoma by increasing venous engorgement resulting in compression of the spinal cord.

\section{References}

1. MacEvilly M, Buggy D (1996) Back pain and pregnancy: a review. Pain 64: 405-414 [Crossref]

2. Antinheimo J, Sankila R, Carpén O, Pukkala E, Sainio M, et al. (2000) Populationbased analysis of sporadic and type 2 neurofibromatosis-associated meningiomas and schwannomas. Neurology 54: 71-76. [Crossref]

3. el-Mahdy W, Kane PJ, Powell MP, Crockard HA (1999) Spinal intradural tumours: Part I--Extramedullary. Br J Neurosurg 13: 550. [Crossref]

4. Seppälä MT, Haltia MJ, Sankila RJ, Jääskeläinen JE, Heiskanen O (1995) Long-term outcome after removal of spinal schwannoma: a clinicopathological study of 187 cases. J Neurosurg 83: 621. [Crossref]

5. Chamberlain MC, Tredway TL (2011) Adult primary intradural spinal cord tumors: a review. Curr Neurol Neurosci Rep 11: 320-328. [Crossref]

6. Zong S, Zeng G, Xiong C, Wei B (2013) Treatment Results in the differential Surgery of Intradural extramedullary Schwannoma of 110 Cases. PloS One 8:e63867. [Crossref] 
Tacken MCT (2016) Unexpected schwannoma unmasked by the physiologic changes of pregnancy

7. Asazuma T, Toyama Y, Maruiwa H, Fujimura Y, Hirabayashi K (2004) Surgical strategy for cervical dumbbell tumors based on a three-dimensional classification. Spine (Phila $\mathrm{Pa}$ 1976) 29: E10-14. [Crossref]

8. Jiang L, Lv Y, Liu XG, Ma QJ, Wei F, et al. (2009) Results of surgical treatment of cervical dumbbell tumors: surgical approach and development of an anatomic classification system. Spine (Phila Pa 1976) 34: 1307-1314. [Crossref]

9. Nadar R, Gragnaniello C, Levy M (2014) Spinal schwannomas. Neurosurgery Tricks of the Trade: Spine and peripheral nerves. Intradural extramedullary tumors. New York: Thieme Publishers.

Copyright: (C2016 Tacken MCT. This is an open-access article distributed under the terms of the Creative Commons Attribution License, which permits unrestricted use, distribution, and reproduction in any medium, provided the original author and source are credited. 